\title{
Hemopericardium after Lung Lobectomy
}

Pardolesi Alessandro $^{1 *}$, Solli Piergiorio ${ }^{3}$ and Spaggiari Lorenzo ${ }^{1,2}$

${ }^{1}$ Division of Thoracic Surgery, European Institute of Oncology, Milan, Italy

2University of Milan, Italy

${ }^{3}$ Division of CardioThoracic Surgery, Papworth Cambridge, UK

\begin{abstract}
Cardiac tamponade after lung resection is a very rare but life-threatening complication. The present case illustrates another of this uncommon complication, wherein the exact mechanism of injury remained undefined. Hemorrhagic cardiac tamponade occurred few hours after left lower lung lobectomy and re-thoracotomy surprisingly revealed that a left coronary artery branch rupture was the cause.
\end{abstract}

Keywords: Cardiac tamponade; Lobectomy; Lung cancer

\section{Introduction}

Cardiac tamponade after lung resection is a very rare but lifethreatening complication. To date only few cases have been described. In the literature the etiology of intrapericardial bleeding was attributable to pre-existing conditions or related to surgical procedure [1-3]. The present case illustrates another of this uncommon complication, wherein the exact mechanism of injury remained undefined. Hemorrhagic cardiac tamponade occurred few hours after left lower lung lobectomy and re-thoracotomy surprisingly revealed that a left coronary artery branch rupture was the cause.

\section{Case Report}

A 61-years-old woman, presented with a $12 \mathrm{~cm}$ diameter left lower lobe tumor with no radiological sings of chest wall or mediastinal infiltration. A fine needle-aspiration biopsy was diagnostic of hamartoma (Figure 1).

The patient underwent a left lateral muscle sparing thoracotomy. Because of the voluminous lesion, dissection of the lower lobe hilar structures was laborious; otherwise the procedure was uneventful, with an estimated blood loss of $200 \mathrm{~mL}$ and a total surgical time of 150 minutes. Transection and suture of inferior pulmonary vein, pulmonary artery branches, left lower bronchus and fissure was achieved by mechanical staplers. Hilar dissection was entirely conducted extrapericardially. Patient was stable in the first post-operative hours, but then her systolic blood pressure decreased suddenly and severely $(60-80 \mathrm{mmHg})$. Chest tube output was minimal at the time $(150 \mathrm{~mL}$ of blood serum fluid since the end of the procedure). The hypotension was unresponsive to fluids and dopamine infusion. Arterial blood gas showed an initial metabolic acidosis, chest X-ray did not reveal any suspicious images, no signs of myocardial ischemia were displayed on electrocardiogram (ECG) and the bedside echocardiogram showed a modest pericardial effusion. An emergent computed tomography scan (CT scan) did not show dissecting aortic aneurysm nor pulmonary embolism but a significant pericardial effusion (Figure 2a). Patient was then transferred to the operating room, nearly three hours after the hypotension event started. We decided for a left re-thoracotomy: No pleural effusion or hemotorax was discovered, nor abnormality in the transected pulmonary hilum structures. The pericardial sac appeared distended and dark-purple colored. A wide pericardial window was performed; after evacuation of $800 \mathrm{~mL}$ of blood a normal systemic blood pressure was restored. The inferior pulmonary vein stump appeared extra-pericardial and intact. We identified a continuous bleeding from a terminal branch of the second obtuse marginal branch of the left circumflex coronary artery (Figure 2b). Hemostasis was achieved with a direct suture with interrupted nonabsorbable polypropylene 5-0 stitches. Bovine

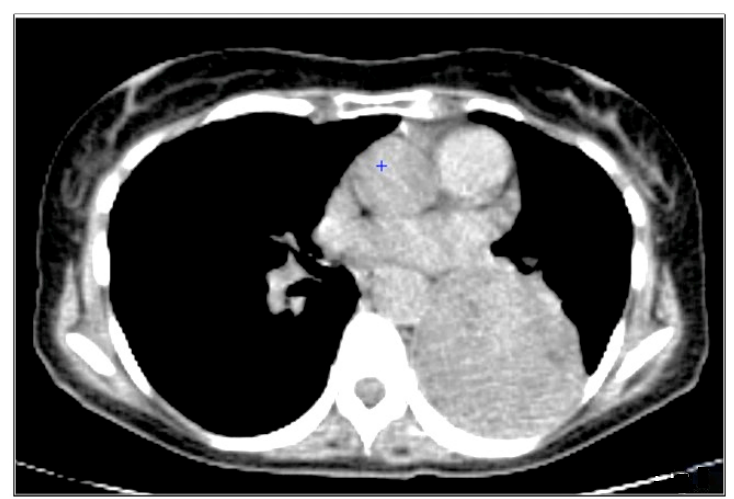

Figure 1: Left lower lobe hamartoma.

pericardium patch was used for the closure of pericardiotomy with interrupted suture. Intraoperative electrocardiogram never displayed signs of myocardial ischemia. Post-operative course was uneventful and patient was discharged ten days after rethoracotomy. During the follow-up period, echocardiogram did not revealed pericardial effusion or myocardial dysfunctions with a normal 6 months postoperative CT scan.

\section{Comment}

Cardiac tamponade is an uncommon complication after lung lobectomy. Some Authors reported cases of intrapericardial bleeding resulting from retraction of the dissected pulmonary vein in the pericardial sac or intraoperative transection of bronchial artery arising from the intrapericardial space [1,2]. Eventhough lymph nodal dissection was not performed because of the benigne nature of the disease, we suspected bleeding could have been originated from the mediastinum or transected lobar hilar structures. We decided to approach the pericardium through a left re-thoracotomy instead of a mediam sternotomy, because of a better exposure of the surgical site.

The etiology of coronary artery rupture is generally related to

*Corresponding author: Alessandro Pardolesi, Division of Thoracic Surgery, European Institute of Oncology, Milan, Via Giuseppe Ripamonti 435, 20141, Italy, Tel: +39 0257489 665; Fax: +39 029437 9218; E-mail: alessandro.pardolesi@ieo.it

Received March 09, 2016; Accepted April 09, 2016; Published April 11, 2016

Citation: Alessandro P, Piergiorio S, Lorenzo S (2016) Hemopericardium after Lung Lobectomy. J Pulm Respir Med 6: 331. doi:10.4172/2161-105X.1000331

Copyright: (c) 2016 Alessandro P, et al. This is an open-access article distributed under the terms of the Creative Commons Attribution License, which permits unrestricted use, distribution, and reproduction in any medium, provided the original author and source are credited. 


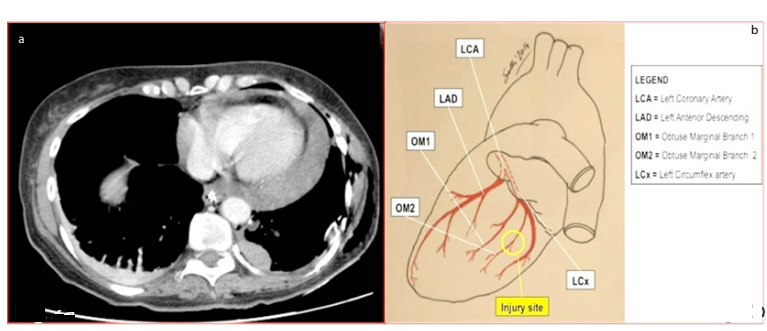

Figure 2: a) Enhanced whole-body computed tomography scan revealing pericardial effusion. b) Coronary artery rupture.

hereditary disorder, such as Ehlers-Danlos syndrome, localized infection, trauma or vascular anomalies [4]. More recently Ozawa et al. [5] reported a case of spontaneous coronary artery rupture causing pericardial tamponade, four days after lung resection.

A revision of patient's medical history did not reveal hereditary disorder, previous traumatic injury or an atherosclerotic disease. Vascular abnormalities or calcifications were not displayed by preoperative CT scan. In the end we could not identify a convincing mechanism of damage. However as the bleeding occurred in the early post-operative hours we hypothesized that intraoperative manipulation of the huge tumor may have caused trauma, especially in the case of a preexisting and undetectable vulnerable lesion of the coronary artery or if the long lasting tumor might have had potentially developed adhesions intrapericardially with coronary vessels. A more careful manipulation of the lobe, avoiding rapid compression and decompression on the pericardium, may have reduced the risk of such a rare complication.

The absence of ECG signs of myocardial ischemia in the preoperative time and during surgery suggested attempting a direct closure of the artery.

Cardiac tamponade caused by coronary artery rupture after lung lobectomy is a very occasional complication, however it should be considered among others when a patient presents with unexplained refractory hypotension in the early postoperative outcome.

\section{References}

1. Tovar EA (1995) Pulmonary resection complicated by abrupt pericardial tamponade. Ann Thorac Surg 60: 1864

2. McLean RH, Parandian BB, Nam MH (1999) Pericardial tamponade: an unusual complication of lobectomy for lung cancer. Ann Thorac Surg 67: 545-546.

3. Miromoto M, Ohashi M, Nobara H, Fukaya Y, Haniuda M, et al. (1991) Rupture of the ascending aorta after surgical resection for lung cancer-a case report. Jpn J Surg 21: 476-479.

4. Evans RH, Fresar AG (1996) Spontaneous coronary artery rupture and cardiac tamponade in Ehlers-Danlos syndrome type IV. Int J Cardiol 54: 283-286.

5. Ozawa Y, Ichimura H, Sato T, Matsuzaki K (2013) Cardiac tamponade due to coronary artery rupture after pulmonary resection. Ann Thorac Surg 96: 97-99. 